\title{
Correspondence
}

\section{p73 is dispensable for commitment to neural stem cell fate, but is essential for neural stem cell maintenance and for blocking premature differentiation}

\author{
Cell Death and Differentiation (2013) 20, 368; doi:10.1038/cdd.2012.134; published online 26 October 2012
}

\section{Dear Editor,}

p73 is a p53 homolog with a major homeostatic role in the central nervous system. ${ }^{1-6}$ Global p73 knockout (KO) exhibit defective embryonic and adult neurogenesis associated with cortical thinning, hydrocephalus and hippocampal dysgenesis. ${ }^{1,3}$ In classic 3D-neurosphere assays from embryonic and newborn whole brains and isolated neurogenic niches, and in vivo tracking of adult neural stem cells (NSCs) in mice, p73 was unequivocally established as an essential regulator of NSC survival and renewal. ${ }^{3-5}$ However, the question whether p73 is also required for the birth of NSCs from neuroectoderm in very early CNS development remained unanswered. The neurosphere assay is not capable of answering this question since it selects for already formed NSCs. Thus, we reasoned that the best system to address this question is to examine NSC formation from $\mathrm{p} 73 \mathrm{KO}$ induced pluripotent stem cells (iPSCs), a tractable surrogate of embryonic stem cells.

To this end, we generated p73KO/Nestin-GFP and WT/ Nestin-GFP mice (Nestin-promoter, an NSC marker, drives GFP), ${ }^{3}$ isolated mouse embryo fibroblasts from littermate embryos and reprogrammed them into iPSCs. ${ }^{7}$ We used either three factors (3F; KIf4, Oct3/4 and Sox2) or two factors (2F; Klf4 and Oct3/4, to avoid possible effects from exogenous Sox2, an NSC regulator). Of note, p73 deficiency had no effect on iPSC generation, self-maintenance or pluripotency (data not shown). Individual iPSC colonies were differentiated into NSCs by N2B27 medium. ${ }^{8}$ FACS analysis showed that the percent of Nestin-GFP + cells (i.e., NSCs) rapidly increased between day 0 and day 3 in both p73KO and WT cells (Supplementary Figure S1a). Importantly, the formation of NSCs from p73KO iPSCs was completely normal up to day 3 in 11 3F-iPSC and 5 2F-iPSC clones from three independent litters analyzed multiple times (Supplementary Figure S1a and data not shown). Thus, these data clearly establish that p73 is not required for commitment to the neural fate and NSC formation, but is essential for NSC maintenance. ${ }^{1-6}$

WT iPSCs in N2B27 medium continuously accumulated NSCs over 7 days or showed a slight decline after peaking at day 3-4. However, at day 7 the percentage of p73KO NSCs was reproducibly lower compared with WT (Supplementary Figure S1a). While brain-derived primary p73KO NSCs exhibit some proliferative defects with increased senescence at day $\mathrm{E} 14,{ }^{3,5}$ our iPSC-derived NSCs most likely represent a younger developmental stage. We excluded that the effect at day 7 is related to proliferation, senescence or apoptosis by labeling iPSCs with a plasma-membrane integrating dye and following its dilutional loss (reflecting cell division) in NestinGFP gated cells, and by senescence assays and growth curves over 7 days (Supplementary Figure S1b and data not shown). Instead, by day 7 iPSC-derived p73KO NSCs might have undergone accelerated neuronal differentiation, as previously observed in brain-derived p73KO NSCs. ${ }^{4}$ Indeed, immunofluorescence analysis of the neuronal marker TuJ1 revealed that at day 4 p73KO cultures had more neurons and a higher percentage of neurons with elongated neurites than WT, indicating faster differentiation (Supplementary Figure S1c). By day 6 , when clusters of neurons had formed, faster differentiation was reflected by a smaller Nestin-GFP progenitor pool (Supplementary Figure S1c, iv). Thus, accelerated NSC loss in day $7 \mathrm{p} 73 \mathrm{KO}$ cultures is due to premature exit into differentiation. Subsequently, however, these neurons develop abnormally, producing thinner, shorter and kinked neurites (Supplementary Figure S1c) as previously reported. ${ }^{3,4}$ This iPSC system supports the notion that $p 73$ blocks differentiation of progenitors independently of its pro-proliferative and anti-senescence action in maintaining the NSC niche. While this study reinforces p73 as a powerful factor in brain development, it also emphasizes that proper p73 expression should be monitored when developing neuronal differentiation protocols for iPSCs in the future for regenerative medicine.

\section{Conflict of Interest}

The authors declare no conflict of interest.

Acknowledgements. This work was funded by NCI grant CA93853 (to UMM) and a NIDDK T32 Fellowship (to EMA).

\author{
EM Alexandrova ${ }^{1}$, F Talos $^{1}$ and UM Mol* ${ }^{*, 1}$ \\ 1 Department of Pathology, Stony Brook University, Stony Brook, NY, USA \\ * Corresponding author: UM Moll, Department of Pathology, Stony Brook \\ University, Nichols Road, Stony Brook, NY 11794-8691, USA. \\ Tel: 631444 2459; Fax: 631444 3424; \\ E-mail: Ute.Moll@sbumed.org
}

1. Yang A et al. Nature 2000; 404: 99-103.

2. Killick R et al. Mol Neurobiol 2011; 43: 139-146.

3. Talos F et al. Cell Death Differ 2010; 17: 1816-1829.

4. Gonzalez-Cano L et al. Cell Death Dis 2010; 1: e109.

5. Agostini $\mathrm{M}$ et al. Biochem Biophys Res Commun 2010; 403: 13-17.

6. Fujitani M et al. Curr Biol 2010; 20: 2058-2065.

7. Nemajerova A et al. Cell Death Differ 2012; 19: 1268-1276.

8. Conti L et al. PLoS Biol 2005; 3: e283. 